\section{Desafios na definição da idade gestacional em estudos populacionais sobre parto pré- termo: 0 caso de um estudo em Campina Grande (PB), Brasil}

\section{Challenges in definition of gestational age in population studies on birth preterm: the case of a study in Campina Grande (PB), Brazil}

\author{
Paula Lisiane de Assunção, \\ Hillegonda Maria Dutilh Novaes' \\ Gizelton Pereira Alencar"I \\ Adriana Suely de Oliveira Melo'v,v \\ Marcia Furquim de Almeida"' \\ 'Faculdade de Medicina da Universidade de São Paulo (USP) - São Paulo (SP), Brasil \\ "Departamento de Saúde da Universidade Estadual do Sudoeste da Bahia - \\ Jequié (BA), Brasil \\ "'Faculdade de Saúde Pública da Universidade de São Paulo (USP) - São Paulo \\ (SP), Brasil \\ IVInstituto de Medicina Integral Prof. Fernando Figueira (IMIP) - Recife (PE), \\ Brasil \\ VInstituto de Pesquisa Prof. Joaquim Amorim Neto (IPESQ) - Campina Grande \\ (PB), Brasil
}

Trabalho realizado na Universidade de São Paulo (USP) - São Paulo (SP), Brasil.

Fonte de financiamento: Fundação de Amparo à Pesquisa do Estado de São Paulo (FAPESP) Processo $n^{\circ}$ 2008/10.948/3.

Correspondência: Paula Lisiane de Assunção - R. José Moreira Sobrinho, SN - Jequiezinho - CEP 45200-000 - Jequié (BH), Brasil - E-mail: pllisiane@gmail.com

Conflito de interesse: nada a declarar.

\section{Resumo}

Introdução: A prevalência de nascimento pré-termo vem aumentando nos últimos anos e é atualmente um problema de saúde pública mundial. Em qualquer tipo de estudo epidemiológico, o grande desafio a ser enfrentado no estudo do parto prétermo é a padronização da aferição da idade gestacional (IG) dos nascimentos. Objetivo: Descrever os métodos utilizados para a definição da amostra a ser estudada e seleção dos eventos em estudo caso-controle dos fatores de risco para nascimento pré-termo de partos hospitalares de mães residentes no município de Campina Grande (PB), Brasil. Métodos: $\mathrm{O}$ desenho foi um casocontrole de base populacional, realizado no período de junho de 2008 a maio de 2009. Os casos foram nascidos com menos de 37 semanas gestacionais e os controles com 37 semanas ou mais. A idade gestacional foi definida em semanas utilizando-se critérios de seleção baseados na acurácia da estimativa. Foram realizadas entrevistas com as mães e coleta de registros hospitalares. Resultados: Foram selecionados 341 casos e 424 controles; 13,19\% foram classificados como pré-termos extremos ( $\leq 28$ semanas), $34,87 \%$ como muito pré-termo ( $<33$ semanas) e $65,10 \%$ como pré-termos moderados (33 a 36 semanas gestacionais). Entre os controles, o percentual de nascidos de 37 a 39 semanas foi de 58,02 e 5,90\% foi de nascidos pós-termo ( $>42$ semanas). Conclusão: As estratégias adotadas mostraram-se viáveis, mesmo em um contexto com maiores limitações na obtenção das informações necessárias, dado que o perfil dos pré-termos, na distribuição da IG, se mostrou comparável a estudos com metodologias mais acuradas.

Palavras-chave: idade gestacional; gravidez; nascimento prematuro; fontes de dados; coleta de dados; estudos de casos e controles. 


\section{Abstract}

Introduction: The prevalence of preterm birth has increased in recent years and it is currently a worldwide public health problem. In any epidemiological study, the greatest challenge facing the study of preterm delivery is to standardize the measurement of gestational age births. Objective: To describe the methods used to define the sample under study and selection of events in case-control studies of risk factors for preterm birth in hospital births by mothers living in the city of Campina Grande (PB), Brazil. Methods: The design was a case-control population-based, which was conducted from June 2008 to May 2009. The cases were born at less than 37 weeks of gestation and controls at 37 weeks or more. Gestational age in weeks was defined using selection criteria based on the accuracy of the estimate. Interviews were conducted with mothers and collection of hospital records. Results: It was selected 341 cases and 424 controls, $13.19 \%$ were classified as extremely preterm ( $\leq 28$ weeks), $34.87 \%$ as very preterm ( $<33$ weeks) and $65.10 \%$ as moderate preterm (33 to 36 weeks of gestation). Among controls, the percent of children born 37-39 weeks was 58.02 to $5.90 \%$ was born post term ( $>42$ weeks). Conclusion: The strategies shown to be viable, even in a context with major limitations in obtaining the necessary information, given that the profile of preterm infants, the distribution of gestational age were comparable to studies with more accurate methods.

Keywords: gestational age; pregnancy; pretermature birth; data sources; data collection; case-control studies.

\section{Introdução}

A incidência do nascimento pré-termo ( $<37$ semanas) vem aumentando globalmente nos últimos 20 anos e se constitui em importante problema de saúde pública ${ }^{1,2}$. A estimativa mundial para o ano de 2005 foi de 9,6\% e as maiores taxas foram observadas nos países da América do Norte (10,6\%) e África (11,9\%), sendo a da Europa a menor (6,2\%). Em números absolutos, a prematuridade afeta de forma desproporcional os países em desenvolvimento, principalmente os da Ásia e África ${ }^{3}$.

$\mathrm{O}$ nascimento pré-termo é atualmente uma das principais causas da mortalidade infantil em países desenvolvidos, superando como causa principal de mortalidade neonatal as mortes por outras causas no seu conjunto $^{4,5}$. Em torno de 75 a $80 \%$ das mortes perinatais ocorrem em nascidos pré-termos ${ }^{6}$. Constitui-se também em fator de risco para morbidade neonatal e da primeira infância, $\mathrm{e}$ em longo prazo pode contribuir para distúrbios do neurodesenvolvimento, dificuldades socioemocionais e comportamentais ${ }^{1,7}$, além de afetar o estado de saúde na vida adulta ${ }^{7,8}$ e trazer anualmente aos países um alto custo social e econômico' ${ }^{1}$.

Apesar das várias pesquisas desenvolvidas e do conhecimento acumulado em relação aos determinantes do nascimento pré-termo, ainda não está clara a razão da tendência ao aumento de suas taxas, principalmente em países industrializados como Estados Unidos e Canadá. Para esses países, as hipóteses concentram-se em mudanças nas intervenções obstétricas, como a determinação da idade gestacional pelo exame ultrassonográfico precoce, a indução ao trabalho de parto e a indicação de parto cirúrgico, bem como no aumento das gestações múltiplas resultantes de reprodução assistida ${ }^{9,10}$.

No Brasil os percentuais de aumento dos pré-termos acompanham o panorama mundial. Recente revisão dos estudos de base populacional realizada com dados primários mostrou aumento da prevalência de 4,0 para $12,0 \%$ entre os anos de 1980 para $2000^{11}$, o que ainda requer melhor compreensão dos seus determinantes ${ }^{12,13}$. 
$\mathrm{Na}$ atualidade, com base no entendimento do nascimento pré-termo como de determinação multifatorial, os esforços voltam-se para a produção de conhecimentos que possam também contribuir para práticas clínicas mais efetivas e impacto na saúde da população $0^{1,2}$. No Brasil, são poucas ainda as pesquisas de base populacional e com dados primários sobre fatores de risco para o parto pré-termo, destacando-se aquelas realizadas nos municípios de Pelotas (RS), Ribeirão Preto (SP) e em São Luiz (MA).

A dificuldade para a realização de estudos sobre parto pré-termo de forma mais ampla se deve, em parte, pelos obstáculos enfrentados no uso dos dados do Sistema de Informação de Nascidos Vivos (SINASC), para essa condição, pois, além da subestimação da prematuridade, em geral ${ }^{14}$, a idade gestacional é coletada na Declaração de Nascido Vivo (DN) em intervalos predeterminados e não em semanas completas, prejudicando as análises estatísticas.

Não existem ainda no Brasil informações coletadas por meio de bases de dados padronizados e de registro da atenção obstétrica e neonatal hospitalar com ampla cobertura, como em países desenvolvidos ${ }^{15}$. Nesse sentido, mostram-se ainda muito importantes estudos populacionais específicos, com coleta de dados primários. Os estudos de tipo coorte prospectiva são os mais adequados para o estudo aprofundado e controlado dos fatores associados ao parto pré-termo ${ }^{16}$, mas na fase atual da pesquisa sobre o tema no Brasil, os estudos de tipo caso-controle ainda se constituem em alternativa útil e viável, principalmente na Região Nordeste, dado o seu relativo baixo custo, menor tempo de coleta e possibilidade de gerar hipótese causal, uma vez que é possível estudar uma variedade de fatores de exposição.

Em qualquer tipo de estudo epidemiológico, o grande desafio a ser enfrentado no estudo do parto pré-termo é a padronização da aferição da idade gestacional (IG) dos nascimentos. Não há ainda um método ideal de estimação definitivo para uso em pesquisas; a mensuração da IG deve ser escolhida e justificada pelos propósitos e parâmetros do estudo ${ }^{17}$ ou deve ser resultante de uma combinação de dois métodos estimativos, com as devidas ressalvas para as limitações próprias de cada um ${ }^{18}$.

As estimativas da IG baseadas na data da última menstruação (DUM) ainda são amplamente utilizadas em estudos de base populacional. Análises comparativas entre a DUM e a estimativa clínica, no entanto, mostram que existem altas discordâncias na estimação de IG extremas ${ }^{17,18}$. Essa discordância é sistemática, o que resulta em superestimação dos pré-termos, moderados e extremos, pela estimativa clínica e subestimação dos pós-termos pela DUM. Considera-se que os erros relacionados à DUM podem ser mais aleatórios e menos sistemáticos comparados à estimativa clínica, o que talvez, confira à DUM um uso mais apropriado em análises de estudos populacionais ${ }^{17}$. Por outro lado, a IG determinada pela estimativa clínica parece mais biologicamente plausível ${ }^{18}$. Outra estimativa que vem ganhando importância em pesquisas, quando há acesso à tecnologia durante o pré-natal, é a baseada em ultrassonografia realizada antes da $20^{\mathrm{a}}$ semana de gestação, considerada a mais acurada para determinar a IG. Entretanto, embora o exame ultrassonográfico seja considerado padrão ouro na prática clínica, sendo preferível às estimativas pós-natais ${ }^{1,19}$, questiona-se sua validade para a pesquisa em razão da subestimação da IG (1-2 dias em média) em fetos menores. Pode-se, no entanto, considerar este viés pequeno ao compará-lo com os erros determinados pela DUM, evidenciados pelas taxas de peso ao nascer implausível para a idade gestacional $^{20}$.

O objetivo deste artigo foi descrever os métodos utilizados para a definição da amostra a ser estudada e seleção dos eventos em estudo caso-controle dos fatores de risco para nascimento pré-termo de partos hospitalares de mães residentes no município de Campina Grande (PB), Brasil.

\section{Métodos}

Local de estudo e prevalência de nascimento pré-termo 
Campina Grande é uma cidade de médio porte localizada na mesoregião do Agreste Paraibano, estado da Região Nordeste do Brasil; Região reconhecidamente com características socioeconômicas precárias para a maioria de seus habitantes.

A cidade possui uma área total de 621 $\mathrm{km}^{2}$, desta, $98 \mathrm{~km}^{2}$ corresponde à área urbana e $420 \mathrm{~km}^{2}$ à área rural. Sua população urbana é de 383.764 habitantes, com uma taxa de crescimento estimada em $0,8 \%$ ao ano para o período de 1996 a 2000. Para o ano 2002, estimou-se 102.452 mulheres em idade fértil, o que correspondeu a $53,7 \%$ da população feminina e $28,8 \%$ da população total ${ }^{21}$.

O Sistema Municipal de Saúde do município é composto por instituições públicas, filantrópicas e privadas perfazendo um total de 133 estabelecimentos, distribuídas nos 6 Distritos Sanitários de Saúde que conformam a rede municipal de saúde local. A rede hospitalar é composta por 18 unidades assistenciais, com 2.148 leitos credenciados ao SUS, sendo 343 destinados à obstetrícia. Dos seis hospitais, entre públicos e privados que atualmente prestam assistência ao parto no município, cinco encerram as demandas mais significativas conforme estimativas de 2005. Apenas dois dispõem de unidade de terapia intensiva neonatal.

Segundo o SINASC $^{22}$, a prevalência média de nascimento pré-termo no município para os anos de 2000 a 2006 foi de $8,9 \%$. Em razão da grande variabilidade da prevalência de pré-termos observada de 2000 a 2006 (Tabela 1), o que poderia refletir inconsistência nos registros no SINASC, foi realizada, no período de março a maio de 2007, uma pesquisa exploratória para identificar a prevalência de nascimento pré-termo nos meses de fevereiro, junho e outubro de 2006, nos hospitais que atendem a quase totalidade da demanda para a assistência ao parto em Campina Grande.

A pesquisa exploratória consistiu em um levantamento de dados arquivados de registros hospitalares sobre a ocorrência de nascidos vivos de mães residentes no município de Campina Grande, nos meses mencionados do ano de 2006. As fontes utilizadas para
Tabela 1. Frequência de nascidos vivos e percentual de nascidos pré-termos de mães residentes em Campina Grande (PB), Brasil, 2000-2006

Table 1. Frequency of live births and percentage of preterm births from mothers living in Campina Grande (PB), Brazil, 2000-2006

\begin{tabular}{lcc}
\hline $\begin{array}{l}\text { Ano do } \\
\text { nascimento }\end{array}$ & $\begin{array}{c}\text { Nascidos de mães } \\
\text { residentes }\end{array}$ & $\begin{array}{c}\% \\
\text { pré-termos }\end{array}$ \\
\hline 2000 & 6.730 & 11,8 \\
2001 & 6.751 & 7,3 \\
2002 & 6.869 & 5,3 \\
2003 & 6.413 & 4,1 \\
2004 & 6.251 & 9,5 \\
2005 & 6.242 & 11,2 \\
2006 & 6.179 & 13,4 \\
\hline
\end{tabular}

Fonte: Ministério da Saúde, Brasil. SINASC, $2008^{22}$. Source: Ministry of Health, Brazil. SINASC, $2008^{22}$.

a coleta dos dados foram: a) livro da sala de parto; b) livro de internação; c) livro de berçário; d) prontuário obstétrico e do recémnascido e e) Declaração de Nascido Vivo (em última instância).

Esse levantamento teve como objetivo subsidiar a elaboração do esquema de seleção da amostra e foi essencial para o desenvolvimento de estratégias de coleta de dados, que envolveram número de controles a serem selecionados mensalmente, tempo de coleta e número de pesquisadores necessários para o trabalho de campo. Para tanto, considerou-se ser suficiente a estimativa da prevalência média de três meses escolhidos em diferentes épocas do ano.

A partir do levantamento, foi possível identificar uma prevalência média de nascimento pré-termo igual a 5,5, o que correspondeu a uma média de 35 nascimentos pré-termo/mês. Sendo necessários, portanto, 12 meses para a coleta da amostra calculada de casos e controles.

\section{Delineamento, população e período de estudo}

O estudo foi de base populacional e investigou nascidos vivos hospitalares, de mães residentes no município, no período de junho/2008 a maio/2009. 


\section{Definição da amostra}

A amostra foi calculada no OpenEpi versão 2. Os parâmetros utilizados foram baseados nos resultados preliminares da análise estatística da pesquisa "Fatores de risco para nascimentos pré-termo", desenvolvido em Londrina (PR), ${ }^{23}$ e na precisão da razão de $o d d s$, a partir dos quais se definiu uma seleção de 394 crianças para o grupo de casos (nascimentos prétermos, menos de 37 semanas de gestação) e 394 crianças para o grupo de controle (nascimentos maior ou igual a 37 semanas de gestação), estratificadas por hospital e com partilha proporcional ao número de nascimento de cada unidade. O tamanho da amostra possibilitou obter estimativas estatisticamente significantes $(\alpha=0,05$ e $1-\beta=0,80)$ para razões de odds tão baixas quanto 1,7 , para uma prevalência esperada de exposição no grupo controle de $15 \%$.

\section{Identificação de casos e controles elegíveis}

Por ser um estudo de base populacional, os casos elegíveis foram todos os nascimentos pré-termos hospitalares, incluindo os nascimentos múltiplos, de mães residentes e ocorridos no período de referência. Os controles foram obtidos a partir de uma amostra dos nascidos não pré-termo e estratificada por hospital.

A identificação e a seleção dos casos e controles elegíveis foram realizadas através de busca ativa diária nos hospitais, tendo como instrumento um Cadastro Diário de Nascimentos e como fonte primária o Livro de Registro de Nascimentos de cada hospital. Os nascimentos ocorridos nas últimas 24 horas eram registrados tomando como horário base o meio dia - 12h00. A identificação das mães residentes em Campina Grande e a confirmação da IG foram realizadas através de consulta preliminar nos prontuários das mães ou dos recém-nascidos, conforme protocolo e rotina de cada hospital.

Dos cinco hospitais pesquisados, em apenas um constava registro do método utilizado para o cálculo da IG. Nos demais se observou, em algumas ocasiões, ausência de qualquer informação sobre a duração da gestação em semanas, sendo, portanto, a seleção do caso ou controle elegíveis realizada a partir das classificações "a termo", "pré-termo" ou "pós-termo" registradas.

A seleção dos controles não foi sequencial com o intuito de evitar dois tipos de viés: a) sobre-representação dos nascimentos do hospital de referência para gestantes de risco na amostra de controles, os quais deixariam de ser representativos do conjunto de nascimentos de não pré-termo da população e b) pareamento involuntário de variáveis de casos e controles proporcionado pelo sorteio de controles sequenciais aos casos, uma vez que o hospital de nascimento tende a ter clientela com perfil socioeconômico semelhante, compreendendo variáveis que se constituem em exposições e que foram focos de avaliação.

Com base na distribuição de nascimentos não pré-termos residentes no município entre os cinco hospitais nos meses investigados, estimou-se o número de nascimentos não pré-termos por hospital no período de 12 meses, período previsto para a coleta.

Para encontrar a proporção mensal de nascimento não pré-termo para cada nascimento pré-termo foi levada em consideração a média de nascimentos não pré-termos residentes em Campina Grande nos três meses pesquisados multiplicada por 12 meses, dividida pela média de nascimentos pré-termos multiplicada por 12 meses. Tendo como parâmetro a relação de 11 nascimentos não pré-termo para cada nascimento pré-termo, obteve-se o número estimado de controles a serem sorteados em cada hospital para compor a amostra. O total de controles (475) calculado excedeu ligeiramente o número definido pela amostragem.

O número semanal de controles previsto para cada hospital foi inferior a um por dia. Desta forma, foi realizado para cada hospital um sorteio dos dias do mês em que os dados dos controles deveriam ser coletados. Nos dias previamente selecionados, foram realizados novamente sorteios entre os nascimentos não pré-termo ocorridos no dia em cada um dos cinco hospitais, para os quais foi realizada a entrevista com a mãe e o preenchimento do protocolo hospitalar. 


\section{Estratégia de seleção e definição de casos e controles}

Para a definição de casos e controles considerou-se os registros das estimativas de IG do obstetra e do pediatra e as IG calculadas pela DUM relatada pela mãe e pelo exame ultrassonográfico realizado com menos de 20 semanas gestacionais. Os critérios adotados foram os mesmos observados na pesquisa de Silva (2008), em Londrina ${ }^{23}$. Nesse trabalho, foi observado que a IG estimada pelo obstetra apresentava uma boa concordância com a IG determinada pelo exame de ultrassonografia realizado antes da $20^{\mathrm{a}}$ semana gestacional, considerada como uma medida de maior precisão e cujo uso é preferencialmente aconselhado em estudos epidemiológicos ${ }^{24}$. Os percentuais de concordância encontrados segundo a idade gestacional foram: $100 \%$ entre os nascidos com menos de 27 semanas e entre os nascidos de 32 a 36 e $\geq 37$ e $89,5 \%$ entre os de 28 a 31 semanas.

A partir dessa observação, a seleção final dos casos e controles (Figura 1) foi baseada na definição da IG considerada em semanas e seguindo os seguintes critérios: a) exclusão de recém-nascidos que não apresentassem IG definida em semanas; b) exclusão de todos os recém-nascidos cuja classificação de prétermo ( $<37$ semanas gestacionais) e não prétermo ( $\geq 37$ semanas gestacionais) apresentasse divergência entre as medidas estimadas; c) definição da IG considerando-se em primeiro lugar o exame de ultrassonografia realizado com menos de 20 semanas gestacionais; d) na ausência deste registro, considerou-se em segundo lugar, a IG estimada pelo obstetra; e) na ausência das informações anteriores, foi considerada, em terceiro lugar, a IG calculada a partir da DUM referida pela mãe e f) havendo apenas o registro da IG estimada pelo pediatra, esta era considerada.

\section{Variáveis do estudo}

A variável desfecho foi o nascimento pré-termo. As variáveis independentes foram agrupadas em cinco blocos, conforme modelo teórico baseado em cadeia hierarquizada considerando-se os três níveis hierárquicos: distal, intermediário e proximal ${ }^{25}$ : a) Bloco 1 - características socioeconômicas; b) Bloco 2 - condições pré-concepcionais e história reprodutiva; c) Bloco 3 - características maternas e gestacionais; d) Bloco 4 - intercorrências maternas na gestação; e) Bloco 5 - características fetais.

\section{Instrumentos e procedimentos para coleta de dados}

Os dados sobre as variáveis maternas biológicas, socioeconômicas, reprodutivas, emocionais, obstétricas e de assistência à saúde no pré-natal e parto foram obtidos através de aplicação de questionário formulado e validado por Almeida, Silva e Matsuo $^{23}$, com algumas alterações necessárias ao atendimento das especificidades da população da qual faz parte a amostra que foi estudada. O instrumento foi submetido a um pré-teste em decorrência das referidas modificações. Também foram coletados dados dos prontuários obstétricos e dos recém-nascidos através de protocolo hospitalar ${ }^{23}$.

As entrevistas com as mães foram realizadas após o parto. Em casos em que não foi possível a realização da entrevista ainda no hospital onde ocorreu o parto, devido ao estado físico/emocional da mãe ou alta precoce, por exemplo, foi agendada sua realização no domicílio da mãe. Nessas situações, era preenchida uma ficha com a identificação da mãe, telefones para contato e endereço. Os não pré-termos sorteados (controles), cujas mães recusaram o convite à participação no estudo, foram substituídos pelo nascimento não pré-termo seguinte registrado no Cadastro Diário de Nascimentos.

\section{Treinamento dos pesquisadores de campo}

A equipe de pesquisadores de campo foi composta por graduandos da área de saúde da Universidade Estadual da Paraíba (UEPB) e da Universidade Federal de 


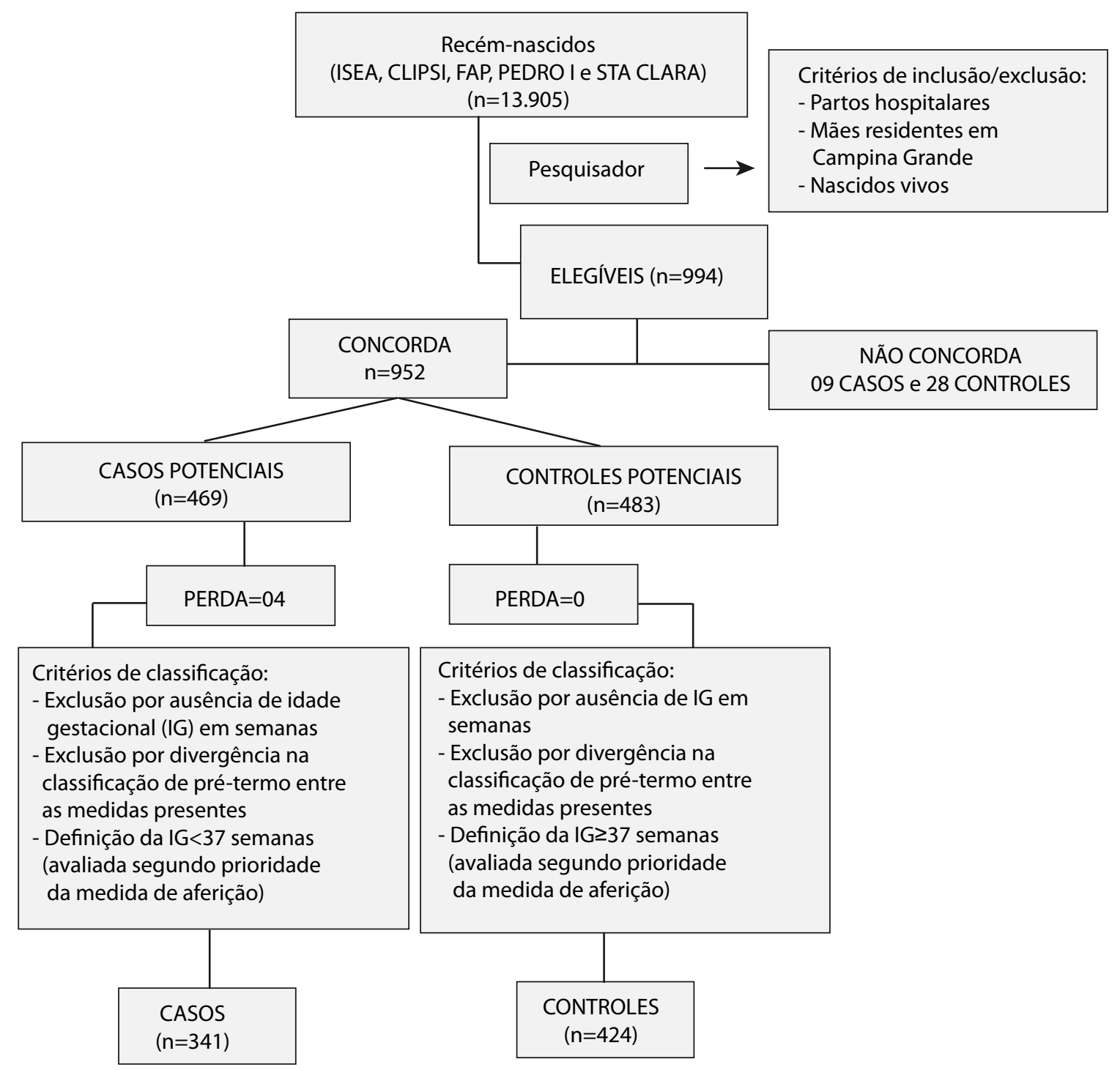

Figura 1. Fluxograma de critérios de seleção

Figure 1. Flowchart of selection criteria

Campina Grande (UFCG) que cursavam no mínimo o segundo ano, selecionados e treinados para este fim. $\mathrm{O}$ treinamento foi realizado por pesquisadores com experiência em trabalho de campo e integrados com as rotinas hospitalares da cidade.

A definição da equipe de trabalho e da logística de campo seguiu-se a um reconhecimento prévio das rotinas dos cincos hospitais que compreenderam o campo de coleta. O treinamento constou de leitura dos instrumentos de coleta de dados orientada por um manual de trabalho de campo, seguida de simulações de entrevistas entre os candidatos e posterior etapa para aplicação dos questionários e protocolos in loco. A seleção foi baseada no desempenho durante o treinamento.

\section{Supervisão, controle de qualidade e trata- mento dos dados}

A coleta foi supervisionada pela pesquisadora responsável localmente pela pesquisa. Os dados coletados eram revisados pelo pesquisador de campo e posteriormente pela supervisora. Foram realizadas reuniões semanais com os pesquisadores 
de campo para checar dados inconsistentes e acompanhar situações adversas àquelas previstas no planejamento da pesquisa. Não sendo possível o esclarecimento da inconsistência de dados durante as reuniões semanais, repetia-se o questionamento à mãe via telefone. Após a crítica dos formulários, os resultados obtidos foram duplamente digitados no Epi Info 3.5.1 para a criação de um banco de dados eletrônico. Ainda em relação ao controle de qualidade da coleta, foram repetidos $5 \%$ de entrevistas e coleta de dados hospitalares. A validação dos bancos foi realizada com o auxílio do programa EpiData 3.1. A seleção final

Tabela 2. Amostra prevista e número e percentual de controles elegíveis segundo hospitais. Campina Grande (PB), Brasil, 2008-2009

Table 2. Expected sample and the number and percentage of eligible controls by hospitals. Campina Grande (PB), Brazil, 2008-2009

\begin{tabular}{lcc}
\hline Hospitais & $\begin{array}{c}\text { Amostra } \\
\text { prevista } \\
\mathrm{n}(\%)\end{array}$ & $\begin{array}{c}\text { Controles } \\
\text { elegíveis } \\
\text { captados } \\
\mathrm{n}(\%)\end{array}$ \\
\hline ISEA & $140(29,5)$ & $123(29,3)$ \\
CLIPSI & $118(24,8)$ & $106(25,2)$ \\
FAP & $113(23,8)$ & $95(22,6)$ \\
Hospital Pedro I & $56(11,8)$ & $49(11,7)$ \\
Sta Clara & $48(10,1)$ & $47(11,2)$ \\
Total & $475(100,0)$ & $420(100,0)$ \\
\hline
\end{tabular}

dos casos e controles foi realizada através do pacote estatístico Stata 8.0.

\section{Considerações éticas}

O projeto de pesquisa foi aprovado pela Comissão de Ética para Análise de Projetos de Pesquisa do Hospital das Clínicas e da Faculdade de Medicina da Universidade de São Paulo/CAPPesq (protocolo de pesquisa $\left.\mathrm{n}^{\circ} 1.207 / 07\right)$. Foram solicitadas autorizações às maternidades para a realização das entrevistas com as mães e para o acesso aos registros e documentações relacionados a estas e aos recém-nascidos. Foi oportunizada a leitura do termo de consentimento livre e esclarecido, elaborado em linguagem compatível, e solicitado o consentimento de participação na pesquisa através da assinatura do referido termo.

\section{Resultados}

O número de nascimentos não pré-termos elegíveis captados (Tabela 2) mostra que foi garantida a distribuição prevista pela amostragem para os controles segundo cada hospital. Desta forma, destaca-se a representatividade da amostra.

Na Figura 1, observa-se o fluxograma dos critérios de seleção utilizados. Em razão da ausência de registro da IG em semanas, bem como da confirmação de divergências entre as diversas fontes de registro observadas em várias ocasiões durante a coleta, a

Tabela 3. Distribuição (número e percentual) de casos e controles selecionados segundo os critérios de prioridade da medida considerada para definição da idade gestacional em semanas. Campina Grande (PB), Brasil, 2008-2009

Table 3. Distribution (number and percentage) of cases and controls selected according to priority criteria for the measurement of gestational age in weeks. Campina Grande (PB), Brazil, 2008-2009

\begin{tabular}{lrrrr}
\hline \multirow{2}{*}{ Fontes definidoras da IG } & \multicolumn{2}{c}{ Casos } & \multicolumn{2}{c}{ Controles } \\
\cline { 2 - 5 } & $\mathrm{n}$ & $\%$ & $\mathrm{n}$ & $\%$ \\
\hline USG $<20$ semanas & 60 & 17,6 & 67 & 15,8 \\
IG estimada pelo obstetra & 195 & 57,2 & 145 & 34,2 \\
DUM (mãe entrevistada) & 74 & 21,7 & 206 & 48,6 \\
IG estimada pelo pediatra & 12 & 3,5 & 6 & 1,4 \\
Total & 341 & 100,0 & 424 & 100,0 \\
\hline
\end{tabular}

IG: idade gestacional; USG: ultrassonografia; DUM: data da última menstruação

IG: gestational age; USG: ultrasound; DUM: last menstrual period 
captação de casos e controles foi expandida em um pouco mais de $10 \%$. Esta estratégia objetivou evitar uma amostra não representativa de casos em virtude das possíveis exclusões.

Ocorreram quatro perdas entre os casos, devido à alta precoce, e nove recusas; destas, sete eram de gestação única e duas de gestação gemelar. Entre os controles observou-se
29 recusas, no entanto, estas foram prontamente substituídas conforme planejamento.

A amostra final obtida após aplicação dos critérios de seleção definidos com base nos registros das IG foi de 341 casos e 424 controles. A Tabela 3 apresenta a distribuição dos casos e controles segundo a fonte da IG considerada. A IG determinada pelo obstetra foi a principal influente para a

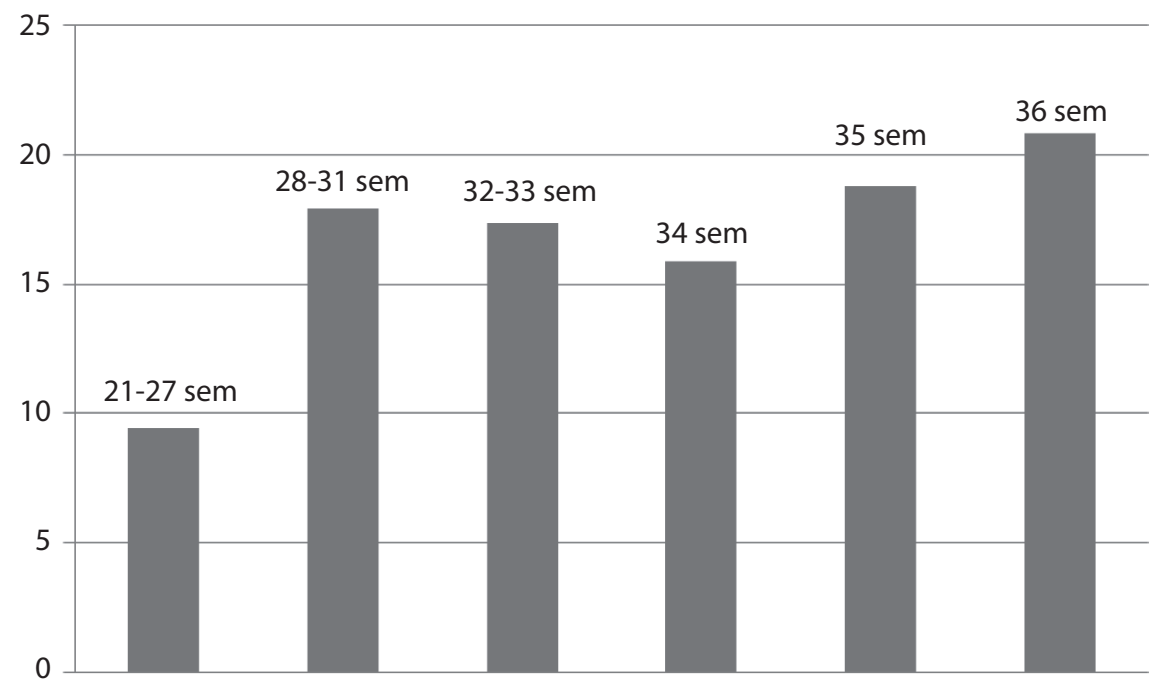

Figura 2. Distribuição (\%) dos casos segundo a idade gestacional em semanas. Nascidos de mães residentes em Campina Grande (PB), Brasil, 2008-2009

Figure 2. Distribution (\%) of cases according to gestational age in weeks. Born from mothers living in Campina Grande (PB), Brazil, 2008-2009

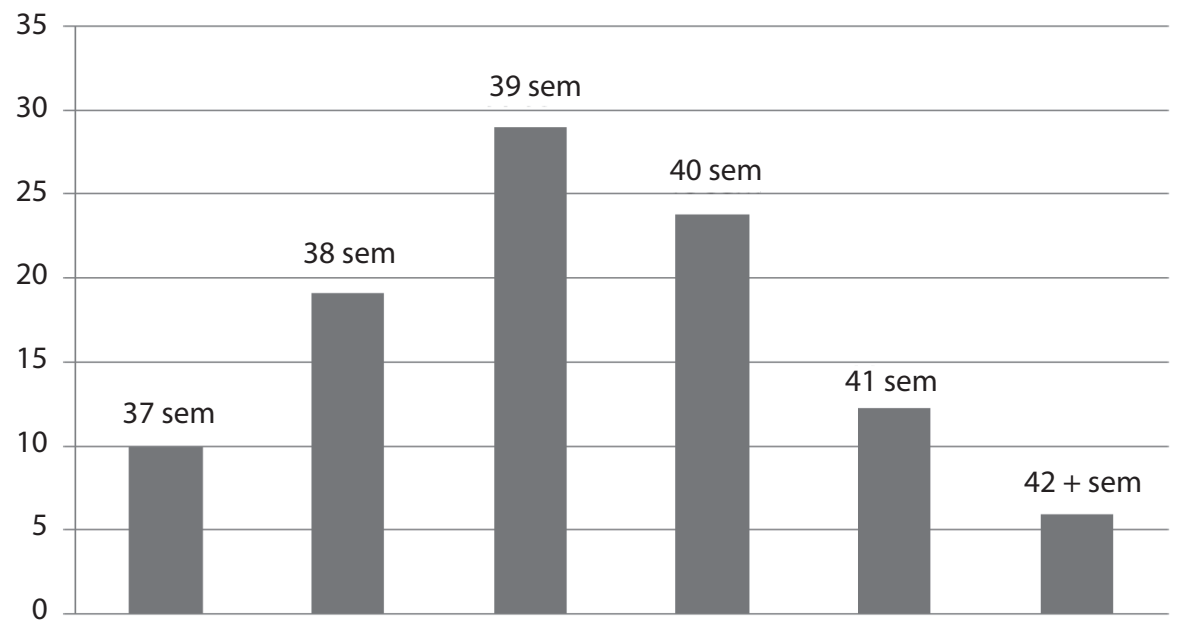

Figura 3. Distribuição (\%) dos controles segundo a idade gestacional em semanas. Nascidos de mães residentes em Campina Grande (PB), Brasil, 2008-2009

Figure 3. Distribution (\%) of controls according to gestational age in weeks. Born to mothers living in Campina Grande (PB), Brazil, 2008-2009 
definição dos casos e a DUM para a definição dos controles.

Dentre os casos, $13,19 \%$ foram classificados como pré-termos extremos $(\leq 28$ semanas), $34,87 \%$ como muito pré-termo (<33 semanas) e $65,10 \%$ como pré-termos moderados (33 a 36 semanas gestacionais) (Figura 2). Quanto à distribuição da IG entre os controles, o percentual de nascidos de 37 a 39 semanas foi de $58,02 \%$ e $5,90 \%$ foi de nascidos pós-termo (>42 semanas) (Figura 3).

\section{Discussão}

Nos estudos que utilizam o método caso-controle, a definição de casos e controles é o alicerce para a validade interna. Nesse contexto, a imprecisão, a discordância entre as medidas e a ausência de registros da IG são alguns dos problemas enfrentados na realização de pesquisas na área de epidemiologia neonatal no Brasil.

Nos últimos anos, no entanto, a viabilidade da realização de pesquisas com dados primários no Brasil vem melhorando com o maior acesso da população ao exame ultrassonográfico antes da $20^{\mathrm{a}}$ semana de gestação no cuidado pré-natal. Em estudo realizado em Londrina, Região Sul, com o mesmo método aqui adotado, cerca de $52 \%$ dos casos e $49 \%$ dos controles foram definidos com base nesse exame. Apesar da significante participação da ultrassonografia na definição dos casos, os autores apontaram a possibilidade de ter ocorrido uma sub-representação relativa de prematuros limítrofes (35 e 36 semanas gestacionais) no estudo, por ser essa a faixa de idade mais afetada pelas divergências entre os métodos de mensuração, resultando em maior número de eventos excluídos $^{26}$.

Nos trabalhos desenvolvidos a partir das coortes de nascimento de Pelotas, também na Região Sul, a principal fonte de definição foi a DUM quando houve adequação com o peso, o comprimento e a circunferência cefálica do recém-nascido, caso contrário, foi utilizado o exame ultrassonográfico realizado no primeiro trimestre ou, na ausência deste, o método clínico de Dubowitz ${ }^{13,27}$.

Entretanto, na realidade aqui estudada, mais pobre, a frequência do registro da IG determinada pela ultrassonografia antes de 20 semanas gestacionais foi baixa; DUM e estimação da IG pelo obstetra ainda são os métodos mais utilizados pelos profissionais de saúde do município. Em relação à IG estimada pela obstetra, a Federação Brasileira das Associações de Ginecologia e Obstetrícia (FEBRASGO) ${ }^{28}$ orienta que a estimativa deve basear-se em primeiro plano na DUM quando é precisa, em contrário deve-se basear: na altura uterina, no líquido amniótico contendo grumos (em presença de amniorrexe) ou na comprovação da gestação compatível com 37 semanas a partir da ultrassonografia realizada antes da $20^{\mathrm{a}}$ semana ou a partir de exame laboratorial ou clínico realizado no início da gestação. Na rotina estudada, no entanto, a anotação da IG estimada pelo obstetra no prontuário não explicitava o critério por ele utilizado.

Para que houvesse uma maior segurança no momento da análise, no que diz respeito à validade interna na alocação de casos e controles, garantindo assim a validade externa na comparação ${ }^{29}$, foram mantidos os critérios de exclusão quando havia divergências entre as estimativas obtidas adotados pelo estudo de Silva ${ }^{23}$.

Portanto, semelhante ao ocorrido em Londrina, pode ter havido uma sub-representação de pré-termos limítrofes entre os casos. O percentual com 35 semanas gestacionais observado foi cerca de 19 e de $21 \%$ para os de 36 semanas (Figura 2); em Londrina, os percentuais observados foram de 20 e $32 \%$, respectivamente ${ }^{23}$. No entanto, ao observar o total de pré-termos tardios $(55,43 \%)$ é possível supor que esta sub-representação possa não ter sido tão significativa (Figura 2), uma vez que em países desenvolvidos as taxas de pré-termos tardios entre os pré-termos variam em torno de 60 a $70 \%$. O percentual de pré- 
termo com menos de 33 semanas foi de $34,8 \%$; a taxa de nascidos nesta faixa de IG em países desenvolvidos situa-se em torno de 28 a $40 \%^{2,9,30}$.

Em relação à distribuição da IG da amostra de controles, percebe-se um maior número de nascidos com 39 semanas, o que vai ao encontro dos achados mais recentes que mostram uma tendência em nível global para a diminuição das taxas de IG de 40 semanas ou mais e um aumento das taxas de IG de 37 a 39 semanas $^{5,9,22}$.

Quanto à seleção dos eventos, vale ressaltar a perda mínima de casos, que foi decorrente das estratégias utilizadas no trabalho de campo, com destaque para a realização de entrevistas no domicílio nas situações de alta hospitalar precoce.

Esses achados demonstram que, apesar dos desafios metodológicos inerentes ao estudo do parto pré-termo, e os cuidados necessários na análise dos dados, as estratégias adotadas mostraram-se viáveis, mesmo em um contexto com maiores limitações na obtenção das informações necessárias, dado que o perfil dos pré-termos, na distribuição da IG, se mostrou comparável a estudos com metodologias mais acuradas.

Além disso, a realização da pesquisa contribuiu para a identificação de problemas na qualidade da DN e nos registros clínicos, informação essa de interesse para os gestores locais. Acredita-se que esta experiência pode ser multiplicada, principalmente em contextos similares em que pesam a inconsistência de dados do SINASC quanto à prevalência de parto pré-termo, bem como os problemas relacionados ao registro da idade gestacional.

\section{Referências}

1. Institute of Medicine, Committee on Undestanding Premature Birth and Assuring healthy Outcomes Board on Health Siences Policy. Preterm Birth: causes, consequences, and prevention.. Washington: National Academies Press; 2007.

2. Goldenberg RL, Culhane JF, Iams JD, Romero R. Epidemiology and causes of preterm birth. Lancet. 2008;371(9606):75-84.

3. Beck S, Wojdyla D, Say L, Betran AP, Merialdi M, Requejo JH, et al. The worldwide incidence of preterm birth: a systematic review of maternal mortality and morbidity. Bull World Health Organ. 2010;88(1):31-8.

4. Lawn JE, Cousens S, Zupan J. 4 million neonatal deaths: when? Where? Why? Lancet. 2005;365(9462):891-900.

5. Martin JA, Hamilton BE, Sutton PD, Ventura SJ, Menacker F, Kirmeyer S, et al. Births: Final Data for 2005. National vital statistics reports; vol. 56 n. 6. Hyattsville, MD: National Center for Health Statistics; 2007.

6. Slattery MM, Morrison JJ. Preterm delivery. Lancet. 2002;360(9344):1489-97.

7. Saigal S, Doyle LW. An overview of mortality and sequelae of preterm birth from infancy to adulthood. Lancet. 2008;371(9608):261-9.

8. Järvelin MR, Sovio U, King V, Lauren L, Xu B, McCarthy MI, et al. Early life factors and blood pressure at age 31 the 1966 Northern Finland Birth Cohort. Hipertension. 2004;44(6):838-46.
9. Davidoff MJ, Dias T, Damus K, Russell R, Bettegowda VR, Dolan S, et al. Changes in the gestational age distribution among U.S. singleton births: Impact on rates of late preterm birth, 1992 to 2002. Semin Perinatol. 2006;30(1):8-15.

10. Ananth CV, Vintzileos AM. Epidemiology of preterm birth and its clinical subtypes. J Matern Fetal and Neonatal Med. 2006;19(12):773-82.

11. Silveira MF, Santos A, Barros AJD, Matijasevich A, Barros FC, Victora CG. Aumento da prematuridade no Brasil: revisão de estudos de base populacional. Rev Saúde Pública. 2008; 42(5):957-64.

12. Barros FC, Victora CG, Barros AJ, Santos IS, Albernaz E, Matijasevich A, et al. The challenge of reducing neonatal mortality in middle-income countries: findings from three Brazilian birth cohorts in 1982, 1993, and 2004. Lancet. 2005;365(9462):847-54.

13. Silveira MF, Victora CG, Barros AJD, SantoS IS, Matijasevich A, Barros FC. Determinants of preterm birth: Pelotas, Rio Grande do Sul State, Brazil, 2004 birth cohort. Cad Saúde Pública. 2010;26(1):185-94.

14. Silveira MF, Santos IS, Matijasevich A, Malta DC, Duarte EC. Nascimentos pré-termo no Brasil entre 1994 e 2005 conforme o Sistema de Informações sobre Nascidos Vivos (SINASC). Cad Saúde Pública. 2009;25(6):1267-75.

15. Rego MAS, França EB, Afonso DCC. Avaliação da qualidade da informação do Sistema de Informação Perinatal (SIP-CLAP/OPAS) para monitoramento da assistência 
perinatal hospitalar, Belo Horizonte, 2004. Rev Bras Saúde Matern Infant. 2009;9(3):275-284.

16. Barros AJ, da Silva dos Santos I, Victora CG, Albernaz EP, Domingues MR, Timm IK, et al. Coorte de nascimentos de Pelotas, 2004: metodologia e descrição. Rev Saúde Pública. 2006;40(3):402-13.

17. Wingate S, Alexander GR, Buekens P, Vahratian A. Comparison of gestational age classifications: date of last menstrual period vs. clinical estimate. Ann Epidemiol. 2007;17(6):425-30.

18. Ananth CV. Menstrual versus clinical estimate of gestational age dating in the United States: temporal trends and variability in indices of perinatal outcomes. Paediatric and Perinatal Epidemiology. 2007;21(Suppl 2):22-30.

19. Allen MC. Assessment of gestational age and neuromaturation. Mental Retard Dev Disabil Res Rev. 2005;11(1):21-33.

20. Yang H, Kramer MS, Platt RW, Blondel B, Bréart G, Morin I, et al. How does early ultrasound scan estimate of gestational age lead to higher rates of preterm birth? Am J Obstet Gynecol. 2002;186(3):433-7.

21. Instituto Brasileiro de Geografia e Estatística. Indicadores sociais mínimos [internet]. Brasil, 2003. [citado $10 \mathrm{dez} 2007$ ]. Disponível em: <http://www.ibge.gov.br>.

22. Ministério da Saúde/SVS - Sistema de Informações sobre Nascidos Vivos (SINASC). Indicadores e Dados Básicos [internet]. Brasil, 2008. [citado 10 jan 2008]. Disponível em: <http://tabnet.datasus.gov.br/cgi/idb2008/matriz.htm>.
23. Silva AMR. Fatores de risco para nascimentos pré-termo no Município de Londrina - Paraná. [tese]. São Paulo: Faculdade de Saúde Pública da Universidade de São Paulo; 2008.

24. Savitz DA, Terry Junior JW, Dole N, Thorp Junior JM, Siega-Riz AM, Herring AH. Comparison of pregnancy dating by last menstrual period, ultrasound scanning, and their combination. Am J Obstet Gynecol. 2002;187(6):1660-6.

25. Victora CG, Huttly SR, Fuchs SC, Olinto MTA. The role of conceptual frameworks in epidemiological analysis: a hierarchical approach. Int J Epidemiol. 1997;26(1):224-7.

26. Silva AMR, Almeida MF, Matsuo T, Soares DA. Fatores de risco para nascimentos pré-termo em Londrina, Paraná, Brasil. Cad. Saúde Pública. 2009;5(10):2125-38.

27. Santos IS, Matijasevich A, Silveira MF, Sclowitz IK, Barros AJ, Victora CG, et al. Associated factors and consequences of late preterm births: results from the 2004 Pelotas birth cohort. Paediatr Perinat Epidemiol. 2008;22(4):350-9.

28. Camano L, Souza E. Manual de Orientação FEBRASGO: Assistência ao Parto e Tocurgia. São Paulo: Ponto; 2002.

29. Reichenheim ME, Moraes CL. Alguns pilares para apreciação da validade em estudos epidemiológicos. Rev Bras Epidemiol. 1998;1(2):131-48.

30. Slattery MM, Geary M, Morrison JJ. Obstetric antecedents for preterm delivery. J Perinat Med. 2008;36(4):306-9.

Recebido em: 06/07/2010

Versão final apresentada em: 18/05/2011

Aprovado em: 04/7/2011 